\title{
Painless foot drop: an unusual acute presentation of new onset type 1 diabetes mellitus
}

\author{
Antonella Corcillo@1, Zoe Kleinaki², Stella Kapnisi@2, Nikolaos Fountoulakis1, \\ Giuseppe Maltese ${ }^{1}$, Stephen M Thomas ${ }^{1}$ and Janaka Karalliedde ${ }^{1}$
}

'Department of Diabetes and Endocrinology, Guy's and St Thomas' NHS Foundation trust, London, UK and School of Cardiovascular Medicine and Sciences, King's College London UK and 'European University Cyprus, School of Medicine, Nicosia, Cyprus
Correspondence should be addressed to A Corcillo or J Karalliedde Email antonella.corcillo@kcl.ac.uk or j.karalliedde@kcl.ac.uk

\section{Summary}

A 26-year-old Caucasian female with no past medical history or family history of auto-immune disease presented to the emergency department with new onset painless left foot drop. A panel of blood tests revealed blood glucose of 49.9 $\mathrm{mmol} / \mathrm{L}$ and raised blood ketone levels. The patient was referred to the diabetes team who made a clinical diagnosis of type 1 diabetes (T1DM) and insulin treatment was initiated. Elevated levels of diabetes auto-antibodies were subsequently detected. Nerve conduction studies demonstrated a left common peroneal nerve lesion with conduction block at the fibular head. After 2 weeks of insulin treatment, a significant improvement of her foot drop was observed and after 8 weeks she was walking normally. The most probable cause of her foot drop was acute diabetic mononeuropathy. To our knowledge, there are no similar cases in adult patients reported in the literature. Our case highlights the importance of physicians being aware of atypical presentation of new onset T1DM.

\section{Learning points:}

- There is an increasing incidence of T1DM with more than half of patients presenting after the age of 20.

- Diabetic peripheral neuropathy can present both acutely and as a mononeuropathy.

- Although rare, clinicians should be aware of mononeuropathy as a presenting symptom of T1DM to avoid delay in the treatment initiation.

- This case highlights an unusual presentation of T1DM and illustrates the importance of the early diagnosis and management of T1DM.

\section{Background}

There is an increasing incidence of T1DM worldwide with more than half of people with newly onset T1DM diagnosed after the age of 20 (1). The most frequent presenting symptoms associated with hyperglycemia are polydipsia, polyuria and weight loss (1). Diabetic neuropathy can present both acutely and as a mononeuropathy. Clinicians need to be aware of unusual signs and symptoms that people with T1DM can present with, to avoid delay in the initiation of insulin treatment. This case highlights an unusual presentation of T1DM and illustrates the importance of the early diagnosis and management of T1DM.

\section{Case presentation}

A 26-year-old female presented to the Emergency Department complaining that her left foot was weak and dragging. These symptoms were preceded by a 4-day history of left calf numbness. A general physical examination was 
recorded as unremarkable with a weight of $52 \mathrm{~kg}(\mathrm{BMI}=17$ $\mathrm{kg} / \mathrm{m}^{2}$ ). In view of the presenting symptoms, she was then referred to the neurology team. A neurological examination was performed revealing reduced power in dorsiflexion of the left ankle (4-/5) with normal sensation and reflexes. No other neurological signs or abnormalities were identified. General physical examination was recorded as unremarkable. A panel of blood tests was performed and the initial laboratory investigations were all within normal values, except for a venous blood glucose of $49.9 \mathrm{mmol} / \mathrm{L}$ and a blood ketones level of $2.2 \mathrm{mmol} / \mathrm{L}$ (normal <0.6). The patient was then referred to the diabetes team who made a clinical diagnosis of T1DM and insulin treatment was initiated.

Following a detailed history recording, it emerged that 6 months before the admission, glycosuria was observed when she attended her primary care practice for a routine appointment. At that time, she did not have any hyperglycemic symptoms and a fasting glucose level was found to be normal. She was a non-smoker, social drinker and denied any current or past use of recreational drugs. There was no family history of diabetes, other autoimmune diseases or hereditary neuropathy. She lived with her partner and was a vegan. Her medical history was unremarkable and she did not take any medications.

\section{Investigation}

Nerve conduction studies demonstrated a left common peroneal nerve lesion with conduction block at the fibular head with no abnormalities in other nerves tested (ulnar or medial cutaneous nerve of the forearm). There was no evidence of a generalized large fiber neuropathy. Diabetes autoantibodies titers were raised: glutamic acid decarboxylase (GAD) autoantibody level was $61.7 \mathrm{U} / \mathrm{mL}$ (normal range: $0.0-3.8 \mathrm{U} / \mathrm{mL}$ ) and tyrosine phosphataserelated islet antigen 2 (IA-2) autoantibody level was $12.5 \mathrm{U} /$ $\mathrm{mL}$ (normal range: $0.0-0.65 \mathrm{U} / \mathrm{mL}$ ). Differential diagnoses for acute mononeuropathy were excluded by performing a panel of other laboratory tests, including serum B12, folic acid, thyroid function, full blood count and serum protein immunoelectrophoresis), which were all normal. A CT scan of the head requested by the neurological team was unremarkable.

\section{Treatment}

A basal bolus insulin regimen with insulin detemir twice a day and premeal insulin aspart was initiated following a detailed review and education provided by the multidisciplinary diabetes team. She had daily phone calls for insulin dose titration and weekly outpatient follow-up. The neurology team did not recommend any additional treatment or investigations.

\section{Outcome and follow-up}

Within 2 weeks of insulin treatment initiation, her foot drop improved significantly and within 8 weeks she was walking normally. She gained $3 \mathrm{~kg}$ and was feeling much better and less tired. Repeat neurological examination did not demonstrate any abnormalities. Her glycemic control was optimized with insulin and she reported well controlled home capillary blood glucose readings. Retinal examination did not demonstrate any evidence of diabetic retinopathy.

\section{Discussion}

People with T1DM have a significant deficiency of insulin due to destruction of pancreatic beta cells. T1DM accounts for $5-10 \%$ of all cases of diabetes mellitus (DM), with its incidence increasing by 2-3\% per year worldwide (1).

Diabetic neuropathy (DN) can present as a symmetric peripheral polyneuropathy (DSPN) and diabetic autonomic neuropathy, which are the most common forms of DN or less frequently. as mononeuropathy, radiculoplexus neuropathy or treatment-induced neuropathy (2). DSPN accounts for about $75 \%$ of all DN and occurs in at least $20 \%$ of people with T1DM after 20 years of disease duration (2). Mononeuropathy in DM may have a sudden onset and most frequently involves the median (5.8\% of all diabetic neuropathies), ulnar (2.1\%), radial $(0.6 \%)$ and common peroneal nerves $(0.1 \%)$ (3). Risk factors for DSPN include older age, longer duration of diabetes, poor glycemic control, hypertension, smoking, height and weight and higher levels of low-density lipoprotein cholesterol and triglycerides (2). After excluding other diagnoses, electrophysiological studies might reveal a slow-down in nerve conduction or a blockage of conduction secondary to a nerve entrapment (2). Our case is unusual as this was an adult patient presenting with an acute peroneal mononeuropathy in the setting of a newly diagnosed T1DM. Several similar cases have already been described in the literature; however, these have been in children $(9,10$ and 14 years old) or in the setting of diabetic ketoacidosis or in people with known diabetes or with bilateral foot drop $(4,5,6,7)$. In 1978, Fraser et al. described the clinical features of 51 cases of mononeuropathy seen in a large outpatient clinic (8). Out of these cases, 37\% occurred within 10 years after the diagnosis of DM, whereas in five cases mononeuropathy was present at the time of DM diagnosis (three lateral popliteal nerves, one abducens nerve and one facial nerve) (8). 
Whereas intensive glycemic control has been demonstrated to be effective for the prevention of DN, spontaneous resolution is usually expected over several months in acute mononeuropathy after exclusion of an entrapment of the nerve requiring surgery of decompression (7). In our case, complete recovery of foot drop was observed after 8 weeks and associated with normalization of glucose levels with insulin treatment.

In conclusion, painless mononeuropathy is a rare presentation of newly diagnosed T1DM. In view of the increasing incidence of T1DM, physicians need to be aware of atypical presentations of diabetes and the importance of considering a diagnosis of diabetes in patients presenting with symptoms and signs of acute mononeuropathy.

\section{Declaration of interest}

The authors declare that there is no conflict of interest that could be perceived as prejudicing the impartiality of the research reported.

\section{Funding}

This research did not receive any specific grant from any funding agency in the public, commercial or not-for-profit sector.

\section{Patient consent}

Written informed consent was obtained from the patient.

\section{Author contribution statement}

$S K$ and $Z K$ wrote first draft which was reviewed by J K, N F, S T and A C who edited and finalized version. N F, G M, S T and J K were involved in patient management.

\section{References}

1 DiMeglio LA, Evans-Molina C \& Oram RA. Type 1 diabetes. Lancet 2018 391 2449-2462. (https://doi.org/10.1016/S0140-6736(18)31320-5)

2 Pop-Busui R, Boulton AJM, Feldman EL, Bril V, Freeman R, Malik RA, Sosenko JM \& Ziegler D. Diabetic neuropathy: a position statement by the American Diabetes Association. Diabetes Care 201740 136-154. (https://doi.org/10.2337/dc16-2042)

3 Cameron NE, Eaton SE, Cotter MA \& Tesfaye S. Vascular factors and metabolic interactions in the pathogenesis of diabetic neuropathy. Diabetologia 200144 1973-1988. (https://doi.org/10.1007/ s001250100001)

4 Kapoor S, Sunaram P, Tziaferi Vaya \& Prasad M. Acute mononeuropathy as a first presentation of type 1 diabetes mellitus. Endocrine Abstracts 201645 20. (https://doi.org/10.1530/endoabs.45.P20)

5 Baszyńska-Wilk M, Wysocka-Mincewicz M, Świercz A, Świderska J, Marszał M \& Szalecki M. Peripheral neuropathy as a complication of diabetic ketoacidosis in a child with newly diagnosed diabetes type 1: a case report. Journal of Clinical Research in Pediatric Endocrinology 201810 289-293. (https://doi.org/10.4274/jcrpe.5374)

6 Rangel MA, Baptista C, Santos F, Real MV, Campos RA \& Leite AL. Acute mononeuropathy in a child with newly diagnosed type 1 diabetes mellitus. Journal of Pediatric Endocrinology and Metabolism 201528 341-344. (https://doi.org/10.1515/jpem-2014-0049)

7 Shahani B \& Spalding JMK. Diabetes mellitus presenting with bilateral foot drop. Lancet 19692 930-931. (https://doi.org/10.1016/ s0140-6736(69)90590-x)

8 Fraser DM, Campbell IW, Ewing DJ \& Clarke BF. Mononeuropathy in diabetes mellitus. Diabetes 197928 96-101. (https://doi.org/10.2337/ diab.28.2.96)

Received in final form 21 January 2021

Accepted 23 February 2021 